\title{
Influencia de la consulta de acogida en diálisis sobre la elección del tratamiento renal sustitutivo
}

\author{
Francisco Cirera Segura \\ Unidad Uro-Nefrológica. H.U. Virgen del Rocío. Sevilla. España
}

\section{Resumen}

\section{Introducción}

Creamos la consulta de acogida en diálisis donde aseguramos la elección del tratamiento renal sustitutivo garantizada por la Ley de autonomía del paciente.

\section{Objetivos}

- Valorar la utilidad de la consulta de acogida para garantizar la elección del tratamiento.

- Analizar la elección del tratamiento según la procedencia del paciente tras pasar por consulta.

\section{Material y método}

Estudio descriptivo prospectivo de un año. Historia clínica y entrevistas como fuentes primarias.

\section{Resultados}

En 2014 iniciaron hemodiálisis 273 pacientes. Pasaron por la consulta de toma de decisiones 175 , de los que fueron incidentes 168.

Analizamos 120 pacientes de ERCA. El 66,7\% realizó elección en ERCA, $100 \%$ en la consulta.

De los 48 pacientes no ERCA, realizaron elección: $3,7 \%$ procedente de otras especialidades, $7,7 \%$ de trasplantados y $100 \%$ de diálisis peritoneal. En la consulta el $100 \%$ hizo elección, pasando del $20 \%$ al $100 \%$ en pacientes no ERCA.

\section{Correspondencia:}

Francisco Cirera Segura

C/ Ángel Ripoll Pastor, 4.6 A. 41006 Sevilla

E-mail:paco.cirera@gmail.com
La hemodiálisis fue el tratamiento más elegido independientemente de la procedencia, excepto en el caso de los pacientes procedentes de diálisis peritoneal. Se aumentó el número de pacientes que eligieron diálisis peritoneal tras el paso por la consulta.

\section{Conclusión}

La consulta de acogida fue eficaz para asegurar que se realizara la elección del tratamiento renal sustitutivo.

Tras el paso por la consulta de acogida y la de toma de decisiones, la procedencia de los pacientes deja de ser importante para realizar la elección del tratamiento. Todos los pacientes eligieron contando con la misma información, aumentando la elección de la diálisis peritoneal.

\section{PALABRAS CLAVE}

- TRATAMIENTO RENAL SUSTITUTIVO

- HEMODIÁLISIS

- CONDUCTA DE ELECCIÓN

Influence of the outpatient nursing reception in dialysis patients in the choice of renal replacement therapy

\section{Abstract}

Introduction: We create the outpatient nursing reception in dialysis patients which ensures the choice of renal replacement treatment, guaranteed by the law of patient autonomy. 


\section{Objectives:}

- To assess the usefulness of the outpatient nursing reception to guarantee the choice of treatment.

- Analyze the choice of treatment according to the origin of the patient after passing through nursing reception.

Material and Methods: A prospective descriptive study of one year. Medical records and interviews were used as primary sources.

Results: In 2014, 273 patients were started hemodialysis treatment. 175 patients went through outpatient nursing reception; 169 were new cases.

We analyzed 120 patients with advanced chronic kidney disease (ACKD). $66.7 \%$ of patients made their choice in ACKD, 100\% in the nursing reception. Of the 48 patients with non ACKD made their choice: $3.7 \%$ from other specialties, $7.7 \%$ of transplant recipients, and $100 \%$ of peritoneal dialysis. In the nursing reception, $100 \%$ of patients made their choice, from $20 \%$ to $100 \%$ in those with non ACKD.

Hemodialysis was the chosen treatment, except for patients derived from peritoneal dialysis. The number of patients who chose peritoneal dialysis after passing through the nursing reception was increased.

Conclusion: The outpatient nursing reception was effective to ensure that the choice of renal replacement therapy was performed.

After passing through outpatient nursing and decisionmaking reception, the source of patients is no longer important for the choice of treatment. All patients chose having the same information, increasing the choice of peritoneal dialysis.

\section{KEYWORDS}

- RENAL REPLACEMENT TREATMENT

- HEMODIALYSIS

- CONDUCT OF CHOICE

\section{Introducción}

Una de las funciones de enfermería que se desarroIla en la consulta de enfermedad renal crónica avanzada (ERCA), es informar y preparar a los pacientes para elegir la modalidad de tratamiento renal sustitutivo (TRS) que se realizará una vez llegado el momento.

Este proceso de información y de elección en pacientes con enfermedad renal crónica constituye un derecho del paciente y es una etapa clave para el tratamiento de la persona que padece dicha enfermedad ya que influirá posteriormente en el estilo y calidad de vida del paciente así como en el de sus cuidadores ${ }^{1}$.

Según la ley 41/2002, de 14 de noviembre², básica reguladora de autonomía del paciente y de derechos y obligaciones en materia de información y documentación clínica, la elección de la modalidad de tratamiento de diálisis, se esté o no en lista de trasplante, tiene que hacerla el paciente en todos los casos.

En nuestro hospital se inició hace dos años, un programa en las consulta ERCA para la toma de decisiones respecto a la modalidad de tratamiento que ha aumentado la incidencia de pacientes en diálisis peritoneal (DP) y ha asegurado la elección de la modalidad TRS de los pacientes que pasan por ella. Sin embargo, y a pesar de los medios que se han articulado en las consultas de ERCA, hasta un $50 \%$ de los pacientes inician tratamiento renal sustitutivo de forma no programada o suboptima ${ }^{3}$, como por ejemplo, los pacientes que retornan otras técnicas como DP, trasplante renal o aquellos que inician TRS de forma urgente o no programada, así como los que no han sido seguidos por un nefrólogo. Estos pacientes no tienen acceso a esta información, ni a realizar la elección de la modalidad de tratamiento ${ }^{3-4}$.

Ante esta situación en el año 2014 creamos la consulta de acogida en diálisis, donde entre otras funciones (como la valoración, revisión y educación sobre los cuidados del acceso vascular, información sobre los cambios en la dieta en hemodiálisis, educación sobre el proceso de la diálisis así como de toda su estancia en la unidad), comprobamos que los pacientes hayan hecho la elección del tratamiento renal sustitutivo y en caso de no haberla hecho, se iniciaba dicho proceso remitiéndose, si fuera necesario, a la consulta de toma de decisiones. 


\section{Objetivos}

Los objetivos de este estudio fueron:

- Valorar la utilidad de la consulta de acogida para garantizar la elección de la modalidad de tratamiento renal sustitutivo.

- Analizar la elección del tratamiento renal sustitutivo según la procedencia del paciente tras pasar por la consulta de acogida.

\section{Material y métodos}

Se realizó un estudio longitudinal descriptivo y prospectivo de un año de duración, desde enero a diciembre de 2014.

Se incluyeron todos los pacientes que iniciaron TRS durante ese año en nuestro hospital, y se estudió la elección de TRS de aquellos que se consideraron enfermos renales crónicos en estadio $\mathrm{V}$, descartando aquellos pacientes que iniciaron tratamiento de forma aguda hasta la resolución de su problema. Se consideraron paciente no incidente aquellos que iniciaron TRS por fracasos renal agudo, cambios temporales de modalidad de tratamiento o pacientes que fueron éxitus antes de 3 meses en la técnica.

Utilizamos como fuente primaria de los datos la historia clínica de los pacientes, así como los obtenidos mediante entrevistas semiestructuradas con los pacientes, realizadas antes de iniciar el TRS y una vez decidida la fecha de su inicio. En aquellos casos en los que se inició el TRS de forma no programada, la entrevista tuvo lugar antes de la segunda sesión.

Se recogieron datos demográficos, epidemiológicos y relacionados con la etiología de la enfermedad renal, así como relacionados con la elección de la técnica: si el paciente había realizado la elección de la modalidad y cuando la había realizado.

Se consideró realizada la elección si constaba en la historia del paciente su paso por la consulta de toma de decisiones 0 si el paciente lo expresaba verbalmente durante la entrevista.

También se recogieron datos sobre la procedencia de los pacientes y la forma de inicio del TRS, así como del
TRS que se realizaba al alta de nuestra unidad y del que se realizaba a los 6 meses del inicio.

El análisis de datos se realizó con el paquete estadístico IBM SPSS Statistics 22.0, estableciendo el nivel de significación en $p<0,05$. Para el estudio de las variables cualitativas usamos frecuencias absolutas y relativas. Y para las cuantitativas utilizamos media y la desviación estándar o la mediana y el rango intercuartílico según correspondiera. Las comparaciones por pares se realizaron mediante la prueba de rangos con signos de Wilcoxon.

\section{Resultados}

En el año 2014 iniciaron tratamiento renal sustitutivo con hemodiálisis un total de 273 pacientes, siendo hombres el $59 \%(n=161)$. La edad media fue de $62,37+15,97$ años, con un $52,7 \%(n=144)$ de los pacientes mayores de 65 años.

En la tabla 1 se describe la etiología de la ERC, siendo el $42,1 \%(n=115)$ diabéticos.
Tabla 1. Etiología de la Enfermedad Renal Crónica*.

\begin{tabular}{l|c|}
\hline & Porcentaje válido y frecuencia \\
\hline No filiada & $24,8 \%(51)$ \\
\hline Diabética & $22,3 \%(46)$ \\
\hline Vascular & $14,1 \%(29)$ \\
\hline Glomerulonefritis & $13,1 \%(27)$ \\
\hline Pielonefritis/Nefropatía Túbulo-Intersticial & $11,2 \%(23)$ \\
\hline Poliquistosis & $6,3 \%(13)$ \\
\hline Sistémicas & $5,8 \%(12)$ \\
\hline Hereditarias & $2,4 \%(5)$ \\
\hline
\end{tabular}

*Códigos EDTA 1994-1995 y su agrupación, extraídos del Informe 2006. Módulo Básico. Subsistema de Insuficiencia Renal Crónica. Servicio Andaluz de Salud. Junta de Andalucía.
Pasaron por la consulta de acogida 175 pacientes. De un total de 168 pacientes crónicos, el 99,4\% ( $n=167)$ fue entrevistado en la consulta, el único que no fue entrevistado $(0,6 \%)$ salió de la unidad durante el mes de Enero, y no se pudo entrevistar en la consulta ya que esta empezó a funcionar formalmente el 1 de febrero de 2014. Los 8 pacientes restantes también fueron considerados crónicos aunque finalmente recuperaron función renal. 


\section{Pacientes no incidentes}

Iniciaron tratamiento sustitutivo en hemodiálisis sin ser incidentes en TRS un total de 105 pacientes, con una edad media de $62,9+15,43$ años, siendo hombres el $55,2 \%(n=58)$, mayores de 65 años un $55,3 \%(n=56)$. El $42,9 \%$ era diabético siendo la etiología diabética también la más frecuente $30,2 \%(n=13)$.

El 33,3\% ( $n=35)$ fueron exitus, el 56,2\% ( $n=59)$ recuperaron la función renal. Optaron por tratamiento conservador el $1 \%(n=1)$ y otro $1 \%(n=1)$ fue retirado de hemodiálisis.

El 6,7\% (n=7) de los pacientes procedían de DP e iniciaron TRS por cambio de técnica temporal, volviendo a su técnica y $2(1,9 \%)$ pacientes trasplantados necesitaron diálisis por un fracaso renal agudo hasta su recuperación.

\section{Pacientes incidentes}

Fueron incidentes en tratamiento renal sustitutivo un total de 168 pacientes de los que estudiamos su elección de modalidad de tratamiento. La edad media fue de 62,04+16,34 años, siendo hombre el $61,3 \%$ $(n=103)$, con un $52,4 \%(n=88)$ de los pacientes mayores de 65 años. El 41,7\% $(n=70)$ era diabético siendo la etiología no filiada la más frecuente $27,6 \%$ $(n=45)$. La estancia media en la Unidad de diálisis fue de $1,12+1,24$ meses.

El $20,2 \%$ de los pacientes iniciaron TRS de forma urgente.

\section{Elección según la procedencia}

En el caso de los 120 pacientes procedentes de la consulta de ERCA, sólo el $66,7 \%(n=80)$ habían realizado la elección de tratamiento antes de pasar por la consulta de acogida. Se analizan otros factores de la elección en la Tabla 2.

Tabla 2. Habían elegido técnica al iniciar TRS desde ERCA.

\begin{tabular}{|lcccc|}
\hline & & Previa & Consulta de Acogida & $\mathbf{p}^{\mathbf{1}}$ \\
\hline \multirow{2}{*}{ Según Tiempo } & $>6$ meses $(n=109)$ & $67,9 \%(n=74)$ & $100 \%(n=109)$ & $<0,001$ \\
& $<6$ meses $(n=11)$ & $54,5 \%(n=6)$ & $100 \%(n=11)$ & 0,025 \\
\hline \multirow{2}{*}{ Según Motivo } & ERC Agudizada $(n=17)$ & $29,4 \%(n=5)$ & $100 \%(n=17)$ & $<0,001$ \\
& ERC Avanzada $(n=103)$ & $72,8 \%(n=75)$ & $100 \%(n=103)$ & 0,001 \\
\hline Totales ERCA & & $66,7 \%(n=80)$ & $100 \%(n=120)$ & $<0,001$ \\
\hline
\end{tabular}

${ }^{1}$ Datos representados porcentaje valido y frecuencia. ${ }^{1}$ Prueba de rangos con signos de Wilcoxon.
De los pacientes procedentes de ERCA, un $14,16 \%$ $(n=17)$ iniciaron TRS por agudización de la ERC y el $9,16 \%(n=11)$ iniciaron TRS antes de 6 meses en la consulta ERCA.

Analizamos si habían realizado la elección de la modalidad de TRS un total de 48 pacientes que no habían pasado por la consulta ERCA pero que fueron incidentes en TRS y sólo el 20,8\% ( $n=10)$ la habían realizado antes de pasar por la consulta de acogida. Tabla 3.

Tabla 3. Habían elegido técnica al iniciar TRS sin pasar por ERCA.

\begin{tabular}{lccc|}
\hline & \multicolumn{1}{c}{ Previa } & $\begin{array}{c}\text { Consulta } \\
\text { de Acogida }\end{array}$ & p \\
\hline No Nefrología $(n=27)$ & $3,7 \%(n=1)$ & $100 \%(n=27)$ & $<0,001^{1}$ \\
\hline DP $(n=8)$ & $100 \%(n=8)$ & $100 \%(n=8)$ & - \\
\hline Trasplante $n=13$ & $7,7 \%(n=1)$ & $100 \%(n=13)$ & $0,001^{1}$ \\
\hline Total N0 ERCA & $20,8 \%(n=10)$ & $100 \%(n=48)$ & $<0,001^{1}$ \\
\hline
\end{tabular}

${ }^{1}$ Datos representados porcentaje valido y frecuencia. ${ }^{1}$ Prueba de rangos con signos de Wilcoxon.

En la tabla 4 se refleja la modalidad elegida por los pacientes según su procedencia así como la modalidad de tratamiento donde se encontraban a los 6 meses.

\section{Elección de los pacientes tras consulta de acogida}

De 78 pacientes que eligieron TRS tras su paso por la consulta de acogida, el $87,17 \%(n=68)$ optaron por la HD, frente al $12,82 \%(n=10)$ que decidieron optar por DP, de los que a la fecha de cierre del estudio 4 estaban en la técnica elegida y cuatro pendiente de paso, los otros dos continuaron en HD por contraindicaciones para la DP.

\section{Discusión}

A pesar de que la ley de autonomía del paciente ${ }^{2}$ indica que la elección del TRS es un derecho del paciente, $y$ nos obliga a los profesionales a ofrecerles la información necesaria, incluso por escrito, para poder realizar dicha elección, existen muchos motivos en la practica diaria que hacen que no se pueda cumplir en todos los casos. 
Tabla 4. Elección, salida y técnica final de los pacientes según su procedencia.

\begin{tabular}{|c|c|c|c|c|c|}
\hline & & ERCA & No Nefrología & DP & Trasplante \\
\hline \multirow[t]{4}{*}{ Elección } & HD & $85 \%(n=102)$ & $77,8 \%(n=21)$ & $50 \%(n=4)$ & $84,6 \%(n=11)$ \\
\hline & DP & $13,3 \%(n=16)$ & $22,2 \%(n=6)$ & $50 \%(n=4)$ & $7,7 \%(n=1)$ \\
\hline & Trasplante & $0,85 \%(n=1)$ & - & - & $7,7 \%(n=1)$ \\
\hline & Conservador & $0,85 \%(n=1)$ & - & - & - \\
\hline \multirow[t]{3}{*}{ Salida } & HD & $94,2 \%(n=113)$ & $88,9 \% \quad(n=24)$ & $50 \%(n=4)$ & $100 \%(n=13)$ \\
\hline & DP & $5 \%(n=6)$ & $11,1 \%(n=3)$ & $50 \%(n=4)$ & - \\
\hline & Trasplante & $0,8 \%(n=1)$ & - & - & - \\
\hline \multirow[t]{3}{*}{ Técnica Final } & HD & $92,5 \%(n=111)$ & $85,2 \%(n=23)$ & $50 \%(n=4)$ & $13 \%(n=100)$ \\
\hline & DP & $6,6 \% \quad(n=8)$ & $14,8, \%(n=4)$ & $50 \%(n=4)$ & - \\
\hline & Trasplante & $0,9 \%(n=1)$ & - & - & - \\
\hline
\end{tabular}

${ }^{1}$ Datos representados porcentaje valido y frecuencia. HD, Hemodiálisis; DP, Diálisis Peritoneal; ERCA, Consulta de enfermedad renal crónica avanzada.

Como medidas para intentar solventar estos problemas, se implantó en nuestro un servicio, la consulta de toma de decisiones, donde a través de un proceso estructurado los pacientes reciben la información necesaria para elegir el TRS, y en los últimos años el numero de pacientes que han pasado por ella ha aumentado progresivamente, lo que ha tenido como resultado un aumento de la elección de la diálisis peritoneal como TRS 5 . Sin embargo, detectamos un elevado número de pacientes que por los circuitos establecidos en nuestro centro, no disponen de la posibilidad de pasar por este proceso.

Por este motivo, creamos la consulta de acogida en diálisis, donde se detecta a los pacientes que no han realizado formalmente la elección del TRS y se derivan a la consulta de toma de decisiones.

La causa principal de que los pacientes no realicen la elección del TRS, es el inicio no programado del mismo. Este incluye a aquellos pacientes que inician el TRS de forma urgente, sin un acceso definitivo o en una técnica no elegida por el paciente3. En nuestro caso, el porcentaje de inicio urgente no fue muy alto, aunque si consideramos la ausencia del acceso vascular definitivo y la elección de la técnica, nos acercamos a las cifras del $50 \%$ que describen algunos autores ${ }^{6}$, de pacientes que inician TRS de manera subóptima.

Este tipo de inicio conlleva un mayor uso de la hemodiálisis, como ocurre en nuestro centro, y además lleva aparejado una peor situación clínica y metabólica, mayor uso de hemodiálisis, mayor morbimortalidad e incremento del gasto sanitario $0^{7-8-9}$.
Incluso en aquellos pacientes procedentes de la consulta ERCA, no alcanzamos mejores cifras como se refiere también en el trabajo de Mendelssohn ${ }^{10}$, ya que la agudización de la propia enfermedad, los problemas para realizar el acceso vascular a tiempo, así como el aumento de la población de estas consultas en los últimos años, hace que disminuir estas cifras de inicio no programado sea muy complicado.

Respecto a la elección de los pacientes, la hemodiálisis sigue siendo mayoritaria, aunque desde la implantación de la consulta de toma de decisiones y de acogida en diálisis, se está aumentando el número de pacientes que eligen DP. En nuestra muestra, de aquellos pacientes que no habían elegido TRS, solo el $12,82 \%$ eligió DP. Aunque parezca una cifra baja, este grupo de pacientes habría iniciado TRS en hemodiálisis, y gracias a la información que se les proporcionó en ambas consultas pudieron elegir libremente la técnica deseada.

Si analizamos la elección de los pacientes que reinician TRS desde trasplante, mayoritariamente eligen hemodiálisis, y aunque uno de ellos eligió diálisis peritoneal, que era su técnica antes del trasplante, a los 6 meses todos estaban en hemodiálisis. Aunque esto ocurre en la mayor parte de programas de trasplantes, los criterios para el inicio del TRS tras el trasplante deberían ser los mismos que para los que inician TRS por primera vez ${ }^{11}$, no justificándose esta distribución de pacientes.

De igual forma, aquellos pacientes procedentes de otras técnicas, bien sea trasplante, diálisis peritoneal 0 aquellos pacientes que no han tenido un seguimiento 
por nefrología y que inician TRS de forma urgente y que no pasan por la consulta ERCA, no siempre se les informa de la posibilidad de elegir el TRS al que deben regresar.

Por último, hemos detectado que a pesar de realizar la elección del TRS, a los seis meses, un gran número de pacientes no está en la técnica que había elegido inicialmente si esta no era hemodiálisis. Esto podría deberse a que una vez que el paciente inicia la técnica, es reacio al cambio.

Como conclusión, podemos afirmar que la consulta de acogida en diálisis fue eficaz para asegurar que se realizara la elección del TRS, ya que conseguimos que el $100 \%$ de los pacientes que iniciaron TRS hubiera hecho su elección.

Tras el paso por la consulta de acogida y la derivación de los pacientes a la consulta de tomas de decisiones, la procedencia de los pacientes deja de ser importante en cuanto a la realización de la elección de TRS, ya que todos los pacientes eligieron contando con la misma información, aumentando la elección de DP.

Recibido: 16 agosto 16

Revisado: 18 agosto 16

Modificado: 28 agosto 16

Aceptado: 31 agosto 16

\section{Bibliografía}

1. Pastor J. L, Julián J.C. Claves del proceso de información y elección de modalidad de diálisis en pacientes con insuficiencia renal crónica. Nefrología. Volumen 1.Suplemento 1. 2015: 15-20.

2. BOE. Ley $41 / 2002$ de 14 de noviembre. 274: 40126-40133.

3. Ruiz-Ferrús R, Pérez-Contreras J. Inicio no programado de diálisis y transferencia de pacientes de hemodiálisis a diálisis peritoneal. Nefrologia Sup Ext 2012;3(3):3-7

4. Celadilla 0, Julve $M$, Vives $A$, De Miguel $M$, Arribas MJ, Cagigal $D$, et al. Evaluación de la información recibida por el paciente que inicia diálisis no programada o procedente de trasplante. Libro de comunicaciones. XXXII Congreso SEDEN 07.

5. Pérez Baena A, Ortega Cuadros C, Cirera Segura F et al. La elección del tratamiento sustitutivo renal del paciente en la consulta de enfermería enfermedad renal crónica avanzada (ERCA). Enferm Nefrol 2015; 18 (3): 34-35.

6. Marrón B, Ortiz A, de Sequera P, Martín-Reyes G, de Arriba G, La- mas JM, et al. Impact of end-stage renal disease care in planned dialysis start and type of renal replacement therapy - a Spanish multicentre experience. Nephrol Dial Transplant 2006;21(Suppl.2):ii51-ii55.

7. Górriz JL, Sancho A, Pallardó LM, Amoedo ML, Martín M, Sanz P, et al. Significado pronóstico de la diálisis programada en pacientes que inician tratamiento sustitutivo renal. Un estudio multicéntrico español. Nefrologia 2002;22(1):49-59.

8. Lameire N, Van Biesen W (1999) The pattern of referral of patients with end- stage renal disease to the nephrologist-a European survey. Nephrology, dialysis, transplantation: official publication of the European Dialysis and Transplant Association - European Renal Association 14 Suppl 6: 16-23.

9. Lameire N, Wauters JP, Teruel JL, Van Biesen W, Vanholder R (2002) An update on the referral pattern of patients with end-stage renal disease. Kidney international Supplement: 27-34.

10. Mendelssohn DC, Curtis B, Yeates K, Langlois $S$, MacRae JM, Se- meniuk LM, et al. Suboptimal initiation of dialysis with and wi- thout early referral to a nephrologist. Nephrol Dial Transplant 2011; 26(9):2959-65.

11. Zarraga S, García G, Teruel JL, Torrente J, et al. Elección de la modalidad de diálisis en la insuficiencia renal avanzada del paciente trasplantado renal. Nefrologia 2009;29(Supl 1):44-8. 